\title{
LOS MARCADORES DE JUVENTUD: LA COMPLEJIDAD DE LAS EDADES
}

\author{
DINA KRAUSKOPF
}

\begin{abstract}
RESUMEN
En los últimos años, en Chile y en las sociedades latinoamericanas en general, el enfoque de juventud se ha visto influido por grandes transformaciones interrelacionadas que dicen relación con los cambios epocales, el modelo económico social imperante, la evolución de los paradigmas de las políticas que han ido modificando el enfoque de juventud. Estas consideraciones llevan a comprender la juventud como la fase biográfica que expresa las transformaciones de la sociedad que están viviendo los sujetos. El presente artículo aborda la problemática de cuáles son los elementos a considerar a fin de identificar a las personas jóvenes en el diseño de investigaciones, programas y políticas. Como equipo investigador del Proyecto Anillo hemos planteado la idea de buscar «marcadores» que permitan organizar la recolección de información, desarrollando principalmente un marcador específico: la edad.

PALABRAS ClAVE: FASE JUVENIL, MARCADORES DE JUVENTUD, CURSO VITAL, DIFERENCIACIÓN IDENTITARIA, CIUDADANÍA JUVENIL, INVISIBILIDAD

Magíster en Psicología Clínica, Universidad Iberoamericana, Costa Rica. Académica Universidad del Desarrollo, Santiago, Chile.

E-Mail: dina.krauskopf@gmail.com.

Este artículo es una re-elaboración de la presentación realizada en el II Seminario Internacional «Juventudes-latitudes-SUR: debates en el Chile contemporáneo», desarrollado en el marco del Proyecto Anillo Juventudes SOC1108. Universidad de Concepción, abril de 2014.
\end{abstract}




\title{
OS MARCADORES DE JUVENTUDE: A COMPLEXIDADE DAS IDADES
}

\begin{abstract}
RESUMO
Nos últimos anos, no Chile e nas sociedades latino-americanas em geral, o enfoque de juventude foi influenciado pelas grandes transformações interrelacionadas que têm relação com as mudanças de época, o modelo econômico social imperante e a evolução dos paradigmas das políticas que modificaram o enfoque de juventude. Estas considerações ajudam a compreender a juventude como a fase biográfica que mostra as transformações da sociedade experimentadas pelos sujeitos. Este artículo aborda a problemática de quais são os elementos para considerar a fim de identificar as pessoas jovens no planejamento de pesquisas, programas e políticas. Como equipe pesquisadora do Projeto Anillo, apresentamos a ideia de procurar "marcadores" que permitam organizar a coleta de informação, desenvolvendo principalmente um marcador específico: a idade.
\end{abstract}

PALAVRAS CHAVE: FASE JUVENIL, MARCADORES DE JUVENTUDE, CURSO VITAL, DIFERENCIAÇÃO IDENTITÁRIA, CIUDADANIA JUVENIL, INVISIBILIDADE

\section{YOUTH MARKERS: COMPLEXITY OF AGES}

\begin{abstract}
During the few last years, the Chilean and Latin American societies have witnessed the several significant interrelated transformations relating to epochal changes, the dominant socioeconomic model and the evolution of the paradigms of the policies influencing on the approach of youth; all these considerations leading to understand youth as the biographical stage expressing the transformations the individuals 'societies are underlying. The present article tackles the problem of the elements to be taken into account in order to identify young people in the design of research, programs and policies. As the Proyecto Anillo's research team, we have stated the need of searching for "markers" that allow to organize the data collection, developing a main specific marker: age.
\end{abstract}

KEY WORDS: JUVENILE STAGE, YOUTH MARKERS, LIFE COURSE, IDENTITARY DIFFERENTIATION, YOUTH CITIZENSHIP, INVISIBILITY 


\section{Procesos Claves de la fase JUVenil}

EN LA FASE JUVENIL se producen procesos claves que absorben el ritmo de los tiempos. El desarrollo intelectual y la maduración sexual se constituyen en recursos y motivaciones para elaborar la diferenciación identitaria y desarrollar la vida social con un replanteamiento de las relaciones con el mundo. Podemos entender este desarrollo como un periodo crucial del curso vital donde los sujetos alcanzan la madurez sexual y cerebral, se apoyan en los recursos psicológicos y sociales que obtuvieron en su crecimiento previo, asumen para sí mismos las funciones que les permiten elaborar su identidad, plantearse un sentido de vida propio $^{1}$ y expresar su actoría en la sociedad (Krauskopf, 2013).

Es la etapa donde con mayor intensidad son cruciales las interacciones entre los recursos personales y grupales con las opciones socio-históricas y características del entorno. La trama del desarrollo juvenil se da con el influjo de la globalización y modernización, ${ }^{2}$ alcanzando un mayor o menor grado de multiculturalidad, con relaciones de género e intergeneracionales en transición y estructuras de oportunidades enraizadas en las condiciones históricas, económicas y políticas de sus sociedades.

El posicionamiento de las juventudes en las sociedades latinoamericanas se ha visto impactado por grandes grupos de transforma-

1 El presente se torna preeminente para darle sentido a la vida de adolescentes y jóvenes. Tradicionalmente se habla del «proyecto» de vida juvenil. Como señala Susana Grunbaum en una comunicación personal, no se trata de un proyecto de vida, sino de alcanzar «una vida con proyectos» (que se van estructurando de acuerdo al sentido de vida).

2 La modernización ha traído una esperanza de vida más prolongada. Se generan nuevos ritmos en los hitos del desarrollo. Un ejemplo de ello es la postergación de la edad del matrimonio y de la procreación, que afecta las formas de conducción de la sexualidad así como las relaciones y metas de muchachos y muchachas. Otro ejemplo lo presenta la permanente readecuación y capacitación requerida para sostener exitosamente la capacidad de logro ocupacional durante todo el ciclo vital, lo que modifica la idea, aún prevalente, de una fase de preparación (adolescencia y juventud) y otra de realización y manejo de conocimientos (adultez) que desarrollamos mas adelante. Adultos y jóvenes requieren incorporar permanentemente nuevos conocimientos y prácticas así como renovar proyectos. 
ciones interrelacionadas como son los cambios epocales, el modelo económico social imperante, la evolución de los paradigmas de las políticas y del enfoque de juventud. (Krauskopf, 2005). Estas consideraciones llevan a comprender la juventud como la fase biográfica que expresa las transformaciones de la sociedad que están viviendo los sujetos (Zarzuri, 2014).

Es frecuente la invisibilidad de la juventud, donde se destaca la omisión de las juventudes rurales y la etnicidad. En este contexto la visibilidad juvenil emerge fácilmente con lo disruptivo: los jóvenes se mencionan poco en lo positivo, pero aparecen cuando sus acciones perturban socialmente. Se brinda importancia a los jóvenes en virtud de problemas designados como su sexualidad, la drogadicción, la delincuencia, la llamada deserción escolar (aun cuando se dé en un sistema expulsor).

Esta ausencia de un claro reconocimiento social de la identidad juvenil es un vacío imprescindible de superar para el desarrollo efectivo del conocimiento sobre sus realidades. La visibilización es importante pues hace parte de las representaciones sociales de la juventud que orientan su inclusión, omisión o exclusión de las acciones públicas. De allí el aporte que pueden hacer los «marcadores de juventud» para apuntar al pleno reconocimiento de las capacidades y derechos de las juventudes junto a las medidas necesarias para su cumplimiento. Sin embargo también este objetivo puede verse vulnerado pues la selección de los marcadores se encuentra influida por diversos factores - entre ellos - la concepción previa que existe sobre las juventudes. Esta concepción es necesario explicitarla, por lo que la sistematizamos a continuación.

\section{ENFOQUES DE JUVENTUD EN LAS POLÍTICAS}

Predominan las políticas que entienden a la «juventud» como un período de preparación y transición a la adultez. Cuando el concepto de transición se utiliza en la juventud como equivalente de transitoriedad —el paso de la niñez a la juventud - implícitamente se niega a los y las jóvenes el reconocimiento como sujetos sociales y se destaca su incompletitud usando el término «inmadurez». Desde el enfoque de preparación para una fase de transición, la juventud es la generación de relevo con escasa participación en la toma de decisiones (Krauskopf, 2003). 
La educación ha sido considerada tradicionalmente como la oferta programática central para el desarrollo de la población joven. El futuro y el modelo del rol adulto, cumplirían la función de eje ordenador de su preparación. La meta es el adulto predeterminado por los estereotipos sociales de desarrollo cumplido. Sin embargo, el adulto contemporáneo no corresponde a identidades fijas ni puede imponerlas a jóvenes que están avizorando nuevos sentidos y horizontes. Con la globalización y la modernización, el conocimiento y la flexibilidad para los aprendizajes pasaron a ser sustantivos y son el punto fuerte de las capacidades juveniles. Ello posiciona de modo distinto el rol y la jerarquía de la experiencia, lo que afecta el modelo adulto para orientar la preparación contemporánea.

La universalidad de la educación es sustentada en un supuesto de homogeneidad que no reconoce el futuro incierto ni incorpora a los estratos pobres de la región en su visión de la juventud. Claramente en los últimos tiempos en el país, el modelo de Educación ha hecho crisis, no solo en la desigualdad evidente en el ámbito económico y social sino en el conocimiento de los derechos y la formación demandada por las personas jóvenes.

La invisibilización de los aportes juveniles facilita su visibilización negativa cuando perturban el orden social. Así se da paso fácilmente a la visión de la juventud como etapa problema con un énfasis estigmatizante. La juventud — que desde el paradigma de preparación era vista como la promesa del futuro- pasa a ser vista como la amenaza del presente. Emergen políticas sectoriales y compensatorias con perspectivas reactivas y reduccionistas para tratar el déficit, el riesgo y la trasgresión juvenil. Este enfoque se mantiene con gran fuerza en la sociedad chilena y da lugar a una percepción generalizadora sobre la juventud a partir de polos sintomáticos y problemáticos, como son la delincuencia, las drogas, la deserción escolar, los embarazos, la violencia, etcétera. Desde esta perspectiva, la causa última de las «patologías» juveniles se identifica en el mismo sujeto juvenil, de ahí que la intervención prioriza la acción sobre él y descuida el contexto. La discriminación por edad que afecta a los sectores jóvenes y la cultura poco participativa, ha sometido a los adolescentes y jóvenes a situaciones difíciles, entre ellas la hostilidad policial. La aceptación de los derechos de las personas jóvenes sufre toda suerte de embates.

La construcción de políticas de actores con el fortalecimiento de las identidades sociales de niños, mujeres, etnias y juventudes —entre 
otras - es relativamente reciente. Es creciente la identificación de los propios jóvenes con lo juvenil, en la definición de sus logros y como interlocutores del Estado de Derecho. La persona joven deja de ser definida desde sus incompletitudes y pasa a ser considerada sujeto de derechos. La construcción de la ciudadanía juvenil se contrapone al predominio de los paradigmas tradicionales mencionados y aun vigentes. Ya no se trata solo de la ciudadanía formal de ejercer el derecho al voto a partir de cierta edad. Se han abierto condiciones que favorecen el desarrollo de iniciativas con las juventudes que buscan consolidar la «ciudadanía juvenil», como lo es la actual Convención Iberoamericana de los Derechos de la Juventud que aún no ha sido reconocida por Chile.

Pero, ¿cuáles son los elementos a tomar en cuenta para identificar a las personas jóvenes en el diseño de investigaciones, programas y políticas? Ante esta cuestión, en las reuniones del equipo investigador del Anillo, surgió la idea de buscar marcadores que permitan organizar la recolección de información. El presente texto procura avanzar en su construcción presentando un conjunto de revisiones y reflexiones sobre cómo entender los marcadores desarrollando principalmente un marcador específico: la edad.

\section{LOS MARCADORES DE JUVENTUD}

El término marcadores se utiliza en diversos ámbitos con fines de almacenar, clasificar, compartir. Los usuarios marcan los recursos que encuentran más útiles. De esta forma se crea un ranking de recursos basado en el criterio de los usuarios que permite ir determinando cuáles son los recursos que proporcionan mayor utilidad. Su acción funcional depende del contexto en que sean utilizados.

Los llamados marcadores sociales son formas que contribuyen a compartir enlaces en Internet con otras personas, lograr enlaces con grupos con preferencias similares o generales en áreas como libros, videos, música, compras, mapas, noticias, etcétera. Con la ayuda de los marcadores sociales se puede guardar y organizar información interesante o importante de forma pública o privada, para luego ser compartida con otros usuarios que mantengan similares intereses mediante enlaces por categorías.

En el caso de los marcadores sociales asociados a internet o intranet se categorizan las palabras asignadas por los usuarios relacionados con el recurso. Esto permite a los usuarios estar al tanto de las 
novedades en una categoría determinada una vez que son incluidas y clasificadas por otros. Los marcadores relacionados con el texto pueden ser: a) ordenadores y estructuradores de la información; b) conectores consecutivos y conectores contraargumentativos; y c) reformuladores explicativos y reformuladores de rectificación que expresan lo que ya se había dicho antes, pero de manera más precisa o más clara.

Los marcadores de juventud pueden aportar estas funciones y cumplir un fin operativo para identificar la población juvenil en el ámbito investigativo. Permiten categorizar la información presentándola de una forma organizada tanto para localizarla como para compartirla. En algunos aspectos pueden semejar a los indicadores y categorías, pero lo que marca la diferencia es el uso que se les pretende dar. Constituyen parte de la metodología para el reconocimiento de lo juvenil en la clasificación base donde se aplicarán los enfoques, ya sea cualitativos o cuantitativos.

Es importante explicitar en qué características juveniles se basan las decisiones de estudios e iniciativas programáticas. Los marcadores pueden ser necesarios instrumentalmente, pero son parte de un proceso en que enfrentan muchas maneras de ser joven, modalidades de trasformación que derivan tanto de las transformaciones de los sujetos de investigación, como de la acumulación de conocimientos sobre ellos. A la vez, en este contexto se requiere homogeneizar para actuar y los marcadores pueden contribuir. Proponemos tipos de marcadores y ejemplos sin pretender ser exhaustivos.

Podemos considerar que son marcadores de transversalidad y diversidad la edad, las características que se manifiestan en relación al género, la pertenencia familiar, cultural, étnica, la residencia geográfica, etcétera.

En la estructura social serían marcadores de la posición el estrato económico-social, la ubicación en el sistema escolar, la inserción laboral, la maternidad y paternidad. Cabe considerar la posición de los jóvenes info-pobres e info-ricos en la llamada sociedad del conocimiento (Balardini, 2003). Entre los marcadores que identifican transiciones claves de las trayectorias tenemos el ingreso a la educación secundaria, a las universidades y los estudios técnicos, el ingreso al mundo laboral, la salida del empleo, del liceo, la salida del hogar, el inicio de vida de pareja, el embarazo, el inicio de vida familiar autónoma, la jefatura de familia.

Es necesario considerar marcadores de acción social como la participación en expresiones culturales, movimientos sociales y políticos. 
Si bien, en este artículo, no desarrollaremos el género como marcador, queremos destacarlo por cuanto constituye un referente plenamente aceptado, pero con frecuencia olvidado en los diseños que sustentan el análisis y las propuestas de la condición juvenil. Las mujeres se mantuvieron en condición de minoridad prácticamente hasta comienzos del siglo XX. En el campo psicológico, se valoriza la adolescencia como un periodo óptimo para el desarrollo pleno de la personalidad comenzándose a hablar de adolescencia femenina y se valoriza la adolescencia como un periodo óptimo para el desarrollo pleno de la personalidad considerando las diferencias entre los sexos.

Los movimientos de mujeres lograron mostrar la importancia de este marcador como elemento de relación social que implica poder, regulación, admisibilidad social, demandando reconocer las diferencias de carácter relacional en las trayectorias, la construcción de los roles de género, y entre ellos, a las masculinidades. Sin embargo, en relación a los análisis de la fase juvenil, el género tiende aun a ser invisibilizado, lo que ocurre a menudo con investigadores del género en relación a lo especifico juvenil.

En este artículo nos referiremos a la edad, un marcador que cumple un papel central en las investigaciones y políticas y que, a la vez, es sumamente cuestionado y debatido.

\section{LAS EDADES}

Ser joven es una condición que se está expandiendo, no solo en cuanto a la edad sino en la representación que la juventud tiene en la sociedad (CEPAL, OIJ, SEGIB, 2008). Gerard Lütte (1991) hace una valiosa revisión de la transformación de los límites etáreos y sus implicaciones. Señala que hasta el siglo II AC en Roma la vida estaba dividida en tres fases: niñez, edad adulta y vejez. No existían la categorías de adolescencia y juventud. ${ }^{3}$ Existía la pubertad fisiológica que conducía a una ceremonia religiosa donde se imponía la toga viril para marcar el paso del niño a la edad adulta. Los hijos púberes (filius familias) podían participar en los comicios, acceder a la ma-

3 Aun hoy predomina el criterio de las tres edades, lo que hace de la vejez la tercera edad. Incluso se ha agregado una cuarta edad, referida a los ancianos mayores. No se contabiliza la fase juvenil. En los tiempos mencionados por Lütte se asimilaban los jóvenes a la adultez. Hoy se asimilan a la niñez. 
gistratura, ingresar a la milicia con los mismos derechos y deberes ciudadanos.

A partir del siglo II AC se postergaron los derechos del púber con limitación a la participación en cargos públicos y se pasó a reconocer la madurez social recién a los veinticinco años. Esto se fundamentó para evitar que el menor se viera involucrado en negocios donde se requiere experiencia. Se genera una nueva clase de edad, en semidependencia de los adultos como continuación de la dependencia infantil que deriva de las condiciones biológicas, la que no existe en la adolescencia-juventud. Se sientan las bases para enfocar a la juventud como una fase de preparación con pérdida de su calidad de sujeto social.

Señala Lütte que tal distinción-oposición entre jóvenes y adultos, coincide con la agravamiento de los contrastes sociales entre las clases y los sexos. Afirma que nunca se ha probado que los privilegios de la adolescencia y la juventud requieran de un periodo de marginación y privación de sus responsabilidades adultas y que ello se da en el contexto de sociedades en que existen claras jerarquías, especializaciones del trabajo y diferencias socioeconómicas. Esto lleva a Lütte a concluir que la adolescencia es «una fase de marginación que deriva de las estructuras sociales fundadas en la desigualdad» (Lütte , 1991:35).

Es relevante el hecho de que hasta el siglo II antes de Cristo en Roma, los jóvenes (hombres) eran considerados adultos, tenían todos los derechos y pasaron a perderlos con argumentos que aún en la época contemporánea son esgrimidos.

Recién en 1989 se inicia un reconocimiento explícito de sus derechos en la Convención Internacional de los Derechos del Niño, donde se fija el término de la niñez en los 18 años y que fue firmada por casi todos los países del mundo. Nótese que los jóvenes quedan subsumidos en el concepto de niño, lo que implica que su desarrollo sexual no es considerado en la Convención. En 1985, la Asamblea General de las Naciones Unidas para el Año Internacional de la Juventud, adoptó el límite de catorce años para definir la niñez y la juventud entre quince y veinticuatro años, inclusive. En esta última categoría se plantea la distinción de los adultos jóvenes, entre los veinte y veinticuatro años. 


\section{TABLA 1: PAÍSES CON ORGANISMOS PÚBLICOS DE JUVENTUD Y EDADES DE LA POBLACIÓN META}

\begin{tabular}{|c|c|c|}
\hline País & ORGANISMO OFICIAL EN JUVENTUD & RANGO ETARIO \\
\hline Anguila & Ministerio de Juventud y Cultura & 15 a 24 años \\
\hline $\begin{array}{l}\text { Antigua y } \\
\text { Barbuda }\end{array}$ & $\begin{array}{l}\text { Ministerio de Educación, Deportes, Juventud y } \\
\text { Asuntos de Género }\end{array}$ & 15 a 24 años \\
\hline Argentina & Dirección Nacional de Juventud & 15 a 29 años \\
\hline Bahamas & Ministerio de Juventud, Deportes y Cultura & 15 a 24 años \\
\hline Barbados & $\begin{array}{l}\text { Ministerio de Familia, Cultura, Deportes y Juventud, } \\
\text { División de Juventud }\end{array}$ & $\begin{array}{l}15 \text { a } 24 \text { años } \\
15 \text { a } 29 \text { años }\end{array}$ \\
\hline Belice & Ministerio Educación y Juventud & 15 a 24 años \\
\hline Bolivia & Viceministerio de Género y Asuntos Generacionales & 15 a 29 años \\
\hline Brasil & Secretaria Nacional de Juventude & 15 a 29 años \\
\hline Chile & Instituto Nacional de la Juventud & 15 a 29 años \\
\hline Colombia & Programa Presidencial Colombia Joven & 14 a 26 años \\
\hline Costa Rica & Viceministerio de Juventud & 12 a 35 años \\
\hline Cuba & Unión de Jóvenes Comunistas de Cuba (UJC) & 15 a 30 años \\
\hline Dominica & Ministerio de Cultura, Juventud y Deportes & 15 a 24 años \\
\hline Ecuador & Ministerio del Poder Popular para la Juventud & 18 a 29 años \\
\hline El Salvador & Instituto Nacional de la Juventud & 14 a 26 años \\
\hline Granada & Ministerio de Juventud y Deportes & 15 a 24 años \\
\hline Guatemala & Consejo Nacional de la Juventud & 14 a 30 años \\
\hline Guyana & Ministerio de Juventud y Deportes & 15 a 24 años \\
\hline Honduras & Instituto Nacional de la Juventud & 12 a 30 años \\
\hline $\begin{array}{l}\text { Islas Vírgenes } \\
\text { Británicas }\end{array}$ & Ministerio de Asuntos de la Juventud y Deportes & 15 a 24 años \\
\hline $\begin{array}{l}\text { Islas Vírgenes } \\
\text { (EE.UU.) }\end{array}$ & División de Niños, Jóvenes y Familias & 15 a 24 años \\
\hline Jamaica & Ministerio de Juventud, Deportes y Cultura & 14 a 24 años \\
\hline México & Instituto Mexicano de la Juventud & 12 a 29 años \\
\hline Nicaragua & Secretaría de Juventud & 18 a 30 años \\
\hline Panamá & Dirección de Juventud & 15 a 29 años \\
\hline Paraguay & Viceministerio de Juventud & 15 a 29 años \\
\hline Perú & Consejo Nacional de la Juventud & 15 a 29 años \\
\hline $\begin{array}{l}\text { República } \\
\text { Dominicana }\end{array}$ & Secretaría de Estado de la Juventud & 15 a 35 años \\
\hline $\begin{array}{l}\text { San Cristóbal } \\
\text { y Nieves }\end{array}$ & $\begin{array}{l}\text { Ministerio de Juventud, Deportes, Tecnología de la } \\
\text { Información y Telecomunicaciones y Correo }\end{array}$ & 15 a 24 años \\
\hline $\begin{array}{l}\text { San Vicente y } \\
\text { Granadinas }\end{array}$ & Ministerio Nacional de la Juventud & 15 a 24 años \\
\hline Santa Lucía & $\begin{array}{l}\text { Ministerio de Transformación Social, Desarrollo de } \\
\text { Recursos Humanos, Juventud y Deportes }\end{array}$ & 15 a 24 años \\
\hline $\begin{array}{l}\text { Trinidad y } \\
\text { Tobago }\end{array}$ & Ministerio de Deportes y Asuntos de la Juventud & 15 a 24 años \\
\hline Uruguay & Instituto Nacional de la Juventud & 14 a 25 años \\
\hline Venezuela & Instituto Nacional del Poder Popular de la Juventud & 18 a 28 años \\
\hline
\end{tabular}

Fuente: UNFPA; CEPAL (2011) «Invertir en juventud. Informe regional de población en juventud en América Latina y el Caribe». 
La Organización Mundial de la Salud (OMS) define la adolescencia entre los diez y diecinueve años once meses - lo que se basa en las edades aproximadas en que se inician las modificaciones sexuales y que marcan la culminación de este crecimiento- y la juventud, entre los quince y veinticuatro años, once meses. ${ }^{4}$ Los códigos derivados de la Convención de los Derechos del Niño, limitan la niñez hasta los doce años y a la adolescencia hasta los dieciocho años, con lo que coinciden con el concepto legal de «menor». La Convención Iberoamericana de los Derechos de los Jóvenes sitúa la juventud entre los dieciocho y veinticuatro años.

Uno de los factores que ha dificultado la construcción de políticas de juventud es el problema de las edades en el ámbito legal. Dado que en el ámbito normativo las personas se consideran adolescentes antes de los dieciocho años y luego adultas, se produce una ambigüedad en el reconocimiento del sujeto juvenil - a veces tratado como adolescente, a veces como adulto-. Ello favorece su invisibilización en la planificación social, va en detrimento del avance en las políticas y establece una pugna entre los defensores del ámbito correspondiente a la niñez y la adolescencia, y los involucrados en la promoción de políticas de juventud (Krauskopf y Mora, 2003). Agreguemos que la edad es también factor de discusión en el ámbito investigativo y que en el transcurso de los tiempos no siempre ha tenido el mismo valor e impacto en las trayectorias.

En las políticas de los países de América Latina y el Caribe (ALC) que abordan el desarrollo de las personas por rangos etarios, se encuentran criterios diversos o contradictorios.

Se puede intentar separar los rangos de edad en la fase juvenil de acuerdo a las transformaciones centrales claramente identificables $^{5}$ que se dan en el desarrollo. ${ }^{6}$ En el ámbito de la salud sexual y reproductiva toman relevancia la edad de la menarquía en las mujeres y las poluciones en los varones, la edad de la primera relación sexual, la edad de la primera unión y la edad del primer embarazo.

4 En el quinquenio de quince a veinte años coinciden las categorías de adolescencia y juventud.

5 Son centrales, el desarrollo intelectual y social, las relaciones intrageneracionales e intergeneracionales, el sentido de vida y la actoría identificados por datos más cualitativos. Se ha comprobado, últimamente, que el desarrollo cerebral continúa hasta aproximadamente los 25 años.

6 Hemos considerado organizar los rangos en quinquenios por cuanto facilitan las comparaciones con las diversas bases de datos. 
En las trayectorias sociales son importantes la edad de término de la escolaridad, la emancipación del hogar y el ingreso al trabajo.

Con estas consideraciones hemos organizado los rangos etarios presentados en el siguiente cuadro, reconociendo que indudablemente se trata de un continuo que no es lineal y sufre modificaciones. ${ }^{7}$

\begin{tabular}{|l|l|}
\hline RANGO ETARIO & DENOMINACIÓN/CARACTERÍSTICAS \\
\hline 10 a 14 años 11 meses & $\begin{array}{l}\text { Preadolescentes, período puberal (pubertad) y comienzo } \\
\text { adolescencia }\end{array}$ \\
\hline 15 a 19 años 11 meses & Adolescencia e inicio de la juventud \\
\hline 20 a 24 años 11 meses & Jóvenes e inicio de la adultez emergente \\
\hline 25 a 29 años 11 meses & Adultez emergente \\
\hline
\end{tabular}

Lütte (1991) plantea que cada vez en más difícil distinguir entre adolescencia y juventud y, por ello, los estudiosos no coinciden en su diferenciación, fases ni en los procesos que sistematizan. También cada vez es más difusa la caracterización de la adultez. Jóvenes y adultos requieren estar constantemente aprendiendo y los proyectos de vida no son constantes.

La categoría reciente de adultez emergente (Arnett, 2010) se identifica a partir del reconocimiento de la posposición cultural a la entrada de roles adultos en ciertos grupos. La autonomía se mantiene basada en una semidependencia. Predomina la exploración de posibles direcciones de vida, del amor y la visión del mundo. Deciden poco sobre el futuro con certeza, pues prima un realismo en las estrategias, toma relevancia el desarrollo de la capacidad de agencia y disposiciones más individualizadas que permiten dar dirección a las trayectorias. El autor les adscribe el rango entre ventiún y veintiocho años de edad.

Los estudios sobre estos jóvenes indican que se consideran adultos cuando aceptan la responsabilidad por sí mismos, toman decisiones independientes y son financieramente independientes. Esto difiere de la categorización tradicional de las decisiones centrales que habitualmente marcaban la adultez en el análisis de trayecto-

7 La pubertad es cada vez más temprana. Además, muchos jóvenes en exclusión, no esperan más allá de los cambios hormonales de la pubertad, para asumir responsabilidades de automantención y mantención familiar. Por otro lado, como señalamos anteriormente, la modernización ha permitido la prolongación de la vida lo cual le da un sentido diferente a las edades y la temporalidad de las secuencias en las trayectorias. 
rias. El fenómeno se presenta selectivamente en jóvenes chilenos, especialmente en los grupos económicamente altos y en el género masculino (PNUD, 2003; Pérez, 2008).

\section{REFLEXIONES FINALES}

Cada grupo etario posee particularidades del desarrollo, derechos específicos, necesidades e intereses que deben identificarse en su singularidad, sin que ello signifique anulación, imposición o exclusión de ningún grupo sobre otro. Se avizora en Chile una población creciente de mayor edad que irá dependiendo de una generación constituida cada vez por menos jóvenes. Estos son el segmento poblacional con mayor potencial para enfrentar con flexibilidad las innovaciones tecnológicas, las transformaciones productivas, los cambios sociales y existenciales. Sin embargo, son también los que enfrentan condiciones insuficientes para efectuar con éxito las transiciones propias de sus trayectorias.

La heterogeneidad de los jóvenes chilenos demanda la selección y adecuación de estrategias para enfrentar criterios diversos o contradictorios tanto en la investigación como en el diseño de programas. Los marcadores de juventud pueden aportar elementos instrumentales para organizar la información requerida. Entre estos se encuentra la edad, que si bien no es un dato suficiente y tiene valores distintos en estratos socioeconómicos, condición de género y culturas, debe sustentar las bases para las acciones estratégicas, configurar el mapa de oportunidades de la juventud y ser parte del sustento de las políticas correspondientes. Su valor dependerá desde dónde y hacia dónde estas se dirigen.

SAN JosÉ (COSTA RICA), DICIEMBRE 2013

RECIBIDO: DICIEMBRE 2013 ACEPTADO: SEPTIEMBRE 2014 


\section{REFERENCIAS BIBLIOGRÁFICAS}

ANETT, JeFFREY JENSEN (2000): «Emerging Adulthood: A Theory of Development from the Late Teens through the Twenties». American Psychologist.

BALARDINNI, SERGIO (2003): «Nuevas tecnologías, ciudadanía y participación». Presentado en Conferencia Regional de Juventud: Las Juventudes en Centroamérica. Retos y Perspectivas. CIRMA, Rockefeller Foundation, Fundación Frederich Ebert. Antigua, Guatemala.

CePAL, OIJ, SEgIB (2008): Juventud y cohesión social: un modelo para armar. Santiago: CEPAL.

KRAUSKOPF, DinA (2013): Adolescencia y educación. San José: Editorial EUNED (tercera edición).

- (2005) «Desafíos en la construcción e implementación de las políticas de juventud en América Latina». Nueva Sociedad N²00. Buenos Aires: Nueva Sociedad.

- (2003). Participación social y desarrollo en la adolescencia. San José: UNFPA (tercera edición).

— y MiNOR MORA (2003): «Condiciones de vida de la juventud centroamericana y el desarrollo de políticas sociales. Evaluación del PRADJAL». Organización Iberoamericana de Juventud.

LUTTE, GERARD (1991): Liberar la adolescencia. La psicología de los jóvenes de hoy. Barcelona: Herder.

PÉREZ, CAROLA (2008): «Dimensions of Emerging Adulthood Inventory». En PÉrez, Cumsille y Martínez: Society for Research on Adolescence. Biennal Meeting Chicago.

PORTOLÉS, JOSÉ (2007): Marcadores del discurso. Barcelona: Ariel.

PNUD (2003): Transformaciones culturales e identidad juvenil en Chile. Santiago: PNUD.

ZARZURI, RAÚL (2014): «Transformaciones de la juventud en los últimos cuarenta años». Ponencia presentada en el II Seminario Internacional Proyecto Anillo Juventud. Concepción, Chile. 